\title{
Tree diversity in sub-montane and lower montane primary rain forests in Central Sulawesi
}

\author{
H. Culmsee ${ }^{1}$, R. Pitopang ${ }^{2}$
}

Key words

Flora Malesiana

Lore Lindu

montane forest

primary forest

rain forest

SE Asia

Sulawesi

tree diversity

\begin{abstract}
The tree diversity of sub-montane and lower montane primary forests is studied in plot-based inventories on two sites in Lore Lindu National Park, Central Sulawesi. Out of 166 species in total, $50 \%$ are new records for Sulawesi $(19 \%)$ or the Central Sulawesi province (31\%). Species richness decreases with altitude. In the submontane forest, the highest Family Importance Values (FIV) are reached by the Lauraceae, Fagaceae, Sapotaceae, Moraceae and Euphorbiaceae. In the lower montane forest, the Fagaceae are of major importance (FIV 71.9), followed at some distance by the Myrtaceae, Elaeocarpaceae and Lauraceae. For each site, a group of important families is identified that is of minor importance or absent on the other site. The comparison of basal area (BA), number of species and FIV with published plot-based studies in sub-montane and lower montane primary forests in Malesia (Borneo, Sulawesi, Papua New Guinea) reveals: 1) with 35.4 and $37.1 \mathrm{~m}^{2} \mathrm{ha}^{-1}$, the BA is comparable to that measured in Borneo and Papua New Guinea, but does not support previous findings of extremely high BA in Sulawesi forests; 2) species richness is comparable to that in Borneo and other Sulawesi forests, but lower than in Papua New Guinea; 3) decrease in diversity with altitude is in accordance with findings in Borneo; 4) in sub-montane forests, the Lauraceae are generally important; the Sulawesi studies are closely related to those from Papua New Guinea; 5) the lower montane forests have the Fagaceae and Myrtaceae as most important families in common.
\end{abstract}

Published on 30 October 2009

\section{INTRODUCTION}

A survey of plant species diversity and endemism across five major Malesian islands has indicated that the island of Sulawesi is intermediate for these measures (Roos et al. 2004). This is remarkable, because one would expect at least high endemism rates due to the isolation of the Wallacean island from the Sunda Shelf during the Quaternary period (Primack \& Corlett 2006). Cannon et al. (2007) pointed out that this mediocrity might be related to the fact that collection rates on the island are among the lowest in Indonesia and to the limited taxonomic study. Hence, additional collections, especially from remote and primary forest areas in Sulawesi, are needed to further our knowledge of the island's plant diversity. Our plot-based tree inventories in primary forests of Lore Lindu National Park, Central Sulawesi, explore the species diversity of sub-montane and lower montane forests. We present a large number of new taxonomic records for Sulawesi or the Central Sulawesi province compared to the Checklist of woody plants of Sulawesi (Keßler et al. 2002).

Especially in montane forests of the Malesian tropics, detailed tree surveys are limited. Quantitative altitudinal transect studies are known from Mt Kinabalu, Borneo (Kitayama 1992, Aiba \& Kitayama 1999, Aiba et al. 2005). A tree diversity study on a one-hectare-plot was carried out by Wright et al. (1997) at 900 m altitude in Papua New Guinea. In Lore Lindu National Park, Central Sulawesi, primary forests were studied at $1100-$ $1200 \mathrm{~m}$ altitude by Kessler et al. (2005). The present study is the first to deal with the primary forest of Sulawesi at different elevations. We aim at identifying the most important tree families

\footnotetext{
Department of Vegetation and Phytodiversity Analysis, Albrecht-von-Haller Institute for Plant Sciences, University of Göttingen, Untere Karspüle 2, 37073 Göttingen, Germany;

e-mail: heike.culmsee@bio.uni-goettingen.de.

2 Department of Biology, Faculty of Mathematics and Natural Sciences, Tadulako University, Palu, Sulawesi Tengah 94118, Indonesia.
}

in our surveyed forests as well as detecting changes in tree family composition between sub-montane and lower montane primary forest sites.

\section{METHODS}

\section{Study area}

The two primary forest study sites are located in Lore Lindu National Park, Central Sulawesi, Indonesia. Most parts of the protected area are covered by upland and montane forests on intermediate soils. The forest condition is good to old growth (Cannon et al. 2007).

The first site is situated in Pono Valley at $1050 \mathrm{~m}$ altitude (S 01²9.6', E $120^{\circ} 03.4^{\prime}$, GC-WGS 84). The habitat is a submontane old growth forest on Ferralsol (FAO 2006) developed on metamorphic rocks in a stable level terrain on a mid-slope. Pono Valley is one of the Sulawesi Throughfall Displacement Experiment test sites hosted by the collaborative research centre SFB 552 (University of Göttingen).

The second site is located in the Bariri Forest at $1400 \mathrm{~m}$ altitude (S $01^{\circ} 39.5^{\prime}, \mathrm{E} 120^{\circ} 10.4^{\prime}$, GC-WGS 84). The habitat is a lower montane old growth forest on Nitisol (FAO 2006) developed on sedimentary substrate on a level plateau. A $70 \mathrm{~m}$ tall meteorological scaffold tower is constructed in the centre of the forest. The forest shows small-scale disturbances close-by the tower caused by the tower construction.

\section{Field sampling}

Plot-based tree inventories were carried out in the period from July to September 2006. Collections were completed in 2007.

Plot size was $40 \times 60 \mathrm{~m}(0.24 \mathrm{ha})$ divided up into a $10 \times 10 \mathrm{~m}$ grid. All trees of diameter at breast height $(\mathrm{dbh}) \geq 10 \mathrm{~cm}$ were surveyed. Within the $10 \times 10 \mathrm{~m}$ grid, $5 \times 5 \mathrm{~m}$-sized subplots were nested (0.06 ha). Therein, understorey trees of dbh 2-9.9 cm

(c) 2009 Nationaal Herbarium Nederland

You are free to share - to copy, distribute and transmit the work, under the following conditions:

$\begin{array}{ll}\text { Attribution: } & \text { You must attribute the work in the manner specified by the author or licensor (but not in any way that suggests that they endorse you or your use of the work) } \\ \text { Non-commercial: } & \text { You may not use this work for commercial purposes. }\end{array}$

Non-commercia:

No derivative works: You may not alter, transform, or build upon this work.

For any reuse or distribution, you must make clear to others the license terms of this work, which can be found at http://creativecommons.org/licenses/by-nc-nd/3.0/legalcode. Any of the above conditions can be waived if you get permission from the copyright holder. Nothing in this license impairs or restricts the author's moral rights. 
were additionally sampled. All trees were permanently tagged, pre-identified, their structural parameters (dbh, total height, trunk height) and their spatial position recorded. In Pono Valley six plots were installed \pm parallel to each other aligned to the Sulawesi Throughfall Displacement Experiment test plots (total area $1.44 \mathrm{ha}$ ). In the Bariri Forest, three plots were set up in a radial arrangement around the meteorological tower avoiding disturbed areas (total area $0.72 \mathrm{ha}$ ).

\section{Tree species identification}

Tree species identification was based upon about 2000 specimens (collection numbers $\mathrm{HC}$ ) collected from tagged trees and supplementary trees in flower or fruit. Specimens were deposited at CEB, GOET, $\mathrm{K}$ and $\mathrm{L}$.

Tree species were identified by $\mathrm{H}$. Culmsee using the collection at the National Herbarium of the Netherlands, University of Leiden branch, as reference and by specialists for Elaeocarpaceae (M.J.E. Coode, K), Moraceae (C.C. Berg, L) and Myristicaceae (W.J.J.O. de Wilde, L). Taxa difficult to identify to species, especially in the Myrtaceae, were distinguished as separate species based on morphology of vegetative characters (leaves, twigs and barks).

\section{Tree diversity analysis}

Species-level presence/absence data related to study site included all tagged trees of $\mathrm{dbh} \geq 10 \mathrm{~cm}$ and were complemented by supplementary species found in the understorey subplots. The assessments as new records for Sulawesi or Central Sulawesi were based on comparison with the Checklist of woody plants of Sulawesi (Keßler et al. 2002).

As the size of the sampled area varied between sites, samplebased rarefaction curves (Gotelli \& Colwell 2001) were calculated using EstimateS v8.0.0 (Colwell 2006) to assess the
Table 1 Basic Pono Valley and Bariri Forest tree inventory metrics.

\begin{tabular}{lcc}
\hline & $\begin{array}{c}\text { Pono Valley } \\
\text { (alt. 1 050 m) }\end{array}$ & $\begin{array}{c}\text { Bariri Forest } \\
\text { (alt. 1 400 m) }\end{array}$ \\
\hline No. of tree families & 42 & 36 \\
No. of tree taxa & 123 & 74 \\
Plot size (in ha) & 1.44 & 0.72 \\
Stem density (trees dbh $\left.\geq 10 \mathrm{~cm}, n \mathrm{ha}^{-1}\right)$ & 520 & 592 \\
Stem density (trees dbh 2-9.9 $\left.\mathrm{cm}^{-1} \mathrm{ha}^{-1}\right)$ & 1767 & 2653 \\
Basal area of trees $\geq 10 \mathrm{~cm} \mathrm{dbh}\left(\mathrm{m}^{2} \mathrm{ha}^{-1}\right)$ & 35.4 & 37.1 \\
Basal area of trees $\geq 2 \mathrm{~cm} \mathrm{dbh}\left(\mathrm{m}^{2} \mathrm{ha}^{-1}\right)$ & 38.8 & 40.9 \\
\hline
\end{tabular}

comparability of species richness per site. Sample units were based on individual counts within the $10 \times 10 \mathrm{~m}$ grid, i.e. 24 samples correspond to one plot ( $0.24 \mathrm{ha})$. The analysis included all trees surveyed ( $\mathrm{dbh} 2-9.9 \mathrm{~cm}$ and $\mathrm{dbh} \geq 10 \mathrm{~cm}$ ).

On the family level, relative frequency (based on the enumeration of individuals) and basal area (based on the dbh measured) were calculated. The Family Importance Value (FIV, Mori et al. 1983) was used to assess the contribution of each family to the stand. FIV combines relative family richness (number of species), relative density (number of individuals) and relative dominance (basal area) into one value. Between-site comparison on the family level took into account trees $\mathrm{dbh} \geq 10 \mathrm{~cm}$.

\section{RESULTS}

\section{Species richness}

The sub-montane forest in Pono Valley (alt. $1050 \mathrm{~m}$ ) accommodates 123 tree taxa assigned to 42 families. The lower montane Bariri Forest (alt. $1400 \mathrm{~m}$ ) is much species poorer with 74 tree taxa out of 36 families (Table 1).

Although the size of the sampled area in the Bariri Forest (0.72 ha) is only half of that in the Pono Valley (1.44 ha), the

Table 2 Species list based on plot-based tree inventories. $\mathrm{P}=$ Pono Valley (alt. $1050 \mathrm{~m}$ ); $\mathrm{B}=$ Bariri Forest (alt. $1400 \mathrm{~m}$ ); $(\mathrm{T})=$ records for trees dbh $\geq 10 \mathrm{~cm}$; $(\mathrm{t})=$ supplementary records for small trees dbh $2-9.9 \mathrm{~cm} ; \mathrm{C}=$ new record for Sulawesi; CC = new record for Central Sulawesi in comparison to Keßler et al. (2002); + = record; +! = new species; $(+)$ = probably a new record.

\begin{tabular}{|c|c|c|c|c|c|c|c|c|c|c|c|c|}
\hline & $\mathrm{P}(\mathrm{T})$ & $P(t)$ & $\mathrm{B}(\mathrm{T}) \mathrm{B}(\mathrm{t})$ & $\mathrm{C}$ & $\mathrm{CC}$ & & $\mathrm{P}(\mathrm{T})$ & $P(t)$ & $\mathrm{B}(\mathrm{T})$ & $\mathrm{B}(\mathrm{t})$ & C & $\mathrm{CC}$ \\
\hline Aceraceae & & & & & & Daphniphyllaceae & & & & & & \\
\hline 1 Acer laurinum Hassk. ex Miq. & + & & + & & & 23 Daphniphyllum papuanum Hallier $\mathrm{f}$. & & & + & & + & \\
\hline Annonaceae & & & & & & Dracaenaceae (Liliaceae) & & & & & & \\
\hline 2 Alphonsea javanica Scheff. & + & & & & & 24 Dracaena angustifolia Roxb. & + & & & & + & \\
\hline 3 Cyathocalyx acuminatus C.B.Rob. & + & & & & + & Ebenaceae & & & & & & \\
\hline 4 Goniothalamus philippinensis Merr. & + & & & + & & 25 Diospyros rumphii Bakh. & & & + & & & + \\
\hline 5 Polyalthia lateriflora King & + & & & & & Elaeocarpaceae & & & & & & \\
\hline 6 Popowia pisocarpa Endl. & + & & & & & 26 Elaeocarpus celebicus Koord. & & & + & & & + \\
\hline Apocynaceae & & & & & & 27 Elaeocarpus culminicola Warb. & & & + & & & + \\
\hline 7 Alstonia spectabilis R.Br. & + & & & & & 28 Elaeocarpus dolichostylus Schltr. & + & & & & & \\
\hline Aquifoliaceae & & & & & & 29 Elaeocarpus erdinii Coode & & & + & & & \\
\hline 8 Ilex cymosa Blume & & & + & & + & 30 Elaeocarpus glaber Blume & + & & & & + & \\
\hline Araliaceae & & & & & & 31 Elaeocarpus luteolignum Coode & & & + & & + & \\
\hline 9 Gastonia serratifolia (Miq.) Philipson & + & & + & + & & 32 Elaeocarpus macropus Warb. ex Knuth & + & & & & & + \\
\hline Arecaceae (Palmae) & & & & & & 33 Elaeocarpus musseri Coode & & + & + & & & \\
\hline 10 Areca vestiaria Giseke & + & & & + & & 34 Elaeocarpus octopetalus Merr. & & & + & & & + \\
\hline 11 Pinanga caesia Blume & + & & & & + & 35 Elaeocarpus sect. Coilopetalum Schltr. & + & & & + & & \\
\hline Asteraceae (Compositae) & & & & & & 36 Elaeocarpus L. sp. 1 & & & + & & & \\
\hline 12 Vernonia arborea Buch.-Ham. & + & & + & & & 37 Elaeocarpus L. sp. 2 & + & & & & & \\
\hline Burseraceae & & & & & & Escalloniaceae (Saxifragaceae) & & & & & & \\
\hline 13 Canarium balsamiferum Willd. & + & & & & & 38 Polyosma celebica Schulze-Menz & & & + & & & + \\
\hline 14 Canarium trigonum H.J.Lam & & & + & & + & 39 Polyosma integrifolia Blume & + & & & & & \\
\hline 15 Santiria apiculata A.W.Benn. & + & & & & + & 40 Polyosma latifolia Schltr. & & & + & & + & \\
\hline Caprifoliaceae & & & & & & Euphorbiaceae & & & & & & \\
\hline 16 Viburnum sambucinum Reinw. ex Blume & & & + & & & 41 Acalypha L. sp. & + & & & & & \\
\hline Chrysobalanaceae & & & & & & 42 Antidesma stipulare Blume & + & & & & & \\
\hline 17 Maranthes corymbosa Blume & & + & & & & 43 Antidesma L. sp. & + & & & & & \\
\hline Clusiaceae (Guttiferae) & & & & & & 44 Aporosa lucida (Miq.) Airy Shaw & + & & & & & + \\
\hline 18 Calophyllum L. sp. & + & & + & + & & 45 Bridelia glauca Blume & + & & & & & + \\
\hline 19 Garcinia dulcis (Roxb.) Kunz & & + & & & + & 46 Drypetes minahassae Pax \& K. Hoffm. & + & & & & & + \\
\hline 20 Garcinia lateriflora Blume & + & & & & & 47 Glochidion lucidum Blume & + & & + & & & + \\
\hline 21 Garcinia L. sp. & + & & & & & 48 Homalanthus populneus Pax & + & & + & & & \\
\hline Cyatheaceae & & & & & & 49 Macaranga allorobinsonii Whitmore & + & & + & & & \\
\hline 22 Cyathea celebica Blume & + & & & + & & 50 Macaranga waturandangii Whitmore & & & + & & & \\
\hline
\end{tabular}


Table 2 (cont.)

\begin{tabular}{|c|c|c|c|c|c|c|c|c|c|c|c|c|c|}
\hline & $P(T)$ & $\mathrm{P}(\mathrm{t})$ & $\mathrm{B}(\mathrm{T})$ & $B(t)$ & $\mathrm{C}$ & $\mathrm{CC}$ & & $P(T)$ & $P(t)$ & $\mathrm{B}(\mathrm{T})$ & $B(t)$ & C & $\mathrm{CC}$ \\
\hline 51 Macaranga Thouars sp. & + & & & & & & 108 Ficus aurita Blume & + & & & & + & \\
\hline 52 Mallotus paniculatus (Geiseler) Airy Shaw & + & & & & & & 109 Ficus calcarata Corner & & + & & & + & \\
\hline 53 Trigonopleura malayana Hook.f. & + & & & & & & 110 Ficus crassiramea (Miq.) Miq. & + & & & & & + \\
\hline Fagaceae & & & & & & & 111 Ficus glandulifera (Wall. ex Miq.) King & & & & + & + & \\
\hline 54 Castanopsis acuminatissima & & & & & & & 112 Ficus schwarzii Koord. & & + & & & & \\
\hline (Blume) Rehder & + & & + & & & & 113 Ficus L. sp. (strangler) & + & & & & & \\
\hline 55 Lithocarpus celebicus (Miq.) Rehder & + & & + & & & & 114 Streblus glaber (Merr.) Corner & & & & & & \\
\hline 56 Lithocarpus elegans (Blume) & & & & & & & subsp. glaber & & & + & & & \\
\hline Hatus. ex Soepadmo & & & + & & + & & Myristicaceae & & & & & & \\
\hline $\begin{array}{l}57 \text { Lithocarpus menadoensis } \\
\text { (Koord.) Soepadmo }\end{array}$ & & & + & & & + & $\begin{array}{l}115 \text { Gymnacranthera farquhariana (Hook.f. \& } \\
\text { Thomson) Warb. var. zippeliana (Miq.) }\end{array}$ & & & & & & \\
\hline Gesneriaceae & & & & & + & & R.T.A.Schouten & + & & & & + & \\
\hline 58 Cyrtandra fasciata H.J.Atkins & & + & & & + & & 116 Horsfieldia costulata (Miq.) Warb. & + & & + & & & + \\
\hline Hamamelidaceae & & & & & + & & 117 Knema stellata Merr. subsp. minahassae & & & & & & \\
\hline 59 Sycopsis dunnii Hemsl. & & & + & & + & & (Warb.) W.J.de Wilde & + & & & & + & \\
\hline Himantandraceae & & & & & & & 118 Myristica simiarum A.DC. subsp. celebica & & & & & & \\
\hline $\begin{array}{l}60 \text { Galbulimima belgraveana } \\
\text { (F.Muell.) Sprague }\end{array}$ & + & & + & & & & $\begin{array}{l}\text { (Miq.) W.J.de Wilde } \\
\text { Myrsinaceae }\end{array}$ & & & + & & & + \\
\hline Icacinaceae & & & & & & & 119 Ardisia forbesii S.Moore & & + & & + & & \\
\hline 61 Platea excelsa Blume var. borneensis & & & & & & & 120 Discocalyx silvestris Holthuis & & + & & & & + \\
\hline (Heine) Sleumer & + & & & & + & & 121 Myrsine porteriana Wall. \& A.DC. & & & & + & + & \\
\hline 62 Platea latifolia Blume & & & + & & + & & Myrtaceae & & & & & & \\
\hline Juglandaceae & & & & & & & 122 Myrtaceae sp. 1 & & & & + & & \\
\hline 63 Engelhardtia rigida Blume & & & + & & & & 123 Myrtaceae sp. 2 & + & & + & & & \\
\hline 64 Engelhardtia serrata Blume & + & & + & & & + & 124 Myrtaceae sp. 3 & & & + & & & \\
\hline Lauraceae & & & & & & & 125 Myrtaceae sp. 4 & + & & & & & \\
\hline 65 Cinnamomum polderi Kosterm. & & & & + & & + & 126 Myrtaceae sp. 5 & + & & + & & & \\
\hline 66 Cinnamomum trichophyllum & & & & & & & 127 Myrtaceae sp. 6 & + & & & & & \\
\hline Quisumb. \& Merr. & + & & & & & & 128 Myrtaceae sp. 7 & + & & & & & \\
\hline 67 Cryptocarya crassinerviopsis Kosterm. & + & & & & & & 129 Myrtaceae sp. 8 & + & & & & & \\
\hline 68 Cryptocarya ferrea Blume & & & + & & & + & 130 Myrtaceae sp. 9 & + & & + & & & \\
\hline 69 Cryptocarya glauca Merr. & & & + & & & + & Oleaceae & 1 & & . & & & \\
\hline 70 Cryptocarya laevigata Blume & + & & & & & + & 132 Chionanthus celebicus Koord. & + & & & & + & \\
\hline 71 Cryptocarya subvelutina Elmer & + & & & + & & + & 133 Chionanthus polygamus (Roxb.) Kiew & + & & + & & $T$ & \\
\hline 72 Endiandra rubescens (Blume) Miq. & + & & & & & + & Pandanaceae & & & + & & & \\
\hline 73 Endiandra sulavesiana Kosterm. & & & + & & & $\begin{array}{l}+ \\
+\end{array}$ & 134 Pandanus L.f. sp. & + & & & & & \\
\hline $\begin{array}{l}74 \text { Endiandra velutina Kosterm. } \\
75 \text { Lindera apoensis Elmer }\end{array}$ & & & + & & & $\begin{array}{l}+ \\
+\end{array}$ & & & & & & & \\
\hline $\begin{array}{l}75 \text { Lindera apoensis Elmer } \\
76 \text { Litsea accedentoides Koord. \& Valeton }\end{array}$ & + & & & & & + & 135 Podocarpus neriifolius D.Don & & & & + & & \\
\hline & + & & $\begin{array}{l}+ \\
+\end{array}$ & & + & + & Proteaceae & & & & & & \\
\hline & + & & + & & & + & 136 Macadamia hildebrandii Steenis & & & + & & & + \\
\hline 79 Litsea grandis Hook.f. & & & + & & & + & Rhizophoraceae & & & & & & \\
\hline 80 Litsea timoriana Span. & + & & & & & & 137 Carallia brachiata (Lour.) Merr. & & + & & & & \\
\hline 81 Neolitsea latifolia (Blume) Moore & + & & & & & + & Rosaceae & & & & & & \\
\hline 82 Persea rimosa Zoll. ex Meisn. & & & + & & & + & 138 Prunus grisea (Blume ex Müll.Berol.) & & & & & & \\
\hline 83 Phoebe grandis (Nees) Merr. & + & & 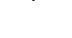 & & & 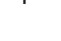 & Kalkman var. grisea & + & & + & & & \\
\hline Leguminosae-Mimosoideae & & & & & & & Rubiaceae & & & & & & \\
\hline 84 Archidendron clypearia (Jack) I.C.Nielsen & + & & & & & + & 139 Diplospora DC. sp. & + & & & & & \\
\hline Magnoliaceae & & & & & & & 140 Gardenia longifolia Vidal & + & & & & & \\
\hline 85 Magnolia liliifera (L.) Baill. var. liliifera & + & & + & & & & 141 Hypobathrum Blume sp. & & + & & & & \\
\hline 86 Magnolia montana (Blume) Figlar \& Noot. & & + & & & + & & 142 Ixora longifolia Valeton & & + & & & + & \\
\hline 87 Magnolia tsiampacca (L.) Figlar \& Noot. & & & & & & & 143 Pavetta celebica Bremek. & & + & & & & \\
\hline var. tsiampacca & + & & & & & & 144 Porterandia celebica Zahid & + & & + & & & + \\
\hline Melastomataceae & & & & & & & 145 Praravinia loconensis Bremek. & & + & & & & + \\
\hline 88 Memecylon paniculatum Jack & + & & & + & & + & 146 Psychotria malayana Jack & + & & & & & + \\
\hline Meliaceae & & & & & & & 147 Timonius minahassae Koord. & + & & & & & \\
\hline 89 Aglaia lancilimba Merr. & + & & & & & + & 148 Urophyllum arboreum Korth. & & & & + & & \\
\hline uamulosa King & + & & & & & & Rutaceae & & & & & & \\
\hline 91 Aglaia tomentosa Teijsm. \& Binn. & + & & & & & & 149 Acronychia pedunculata Miq. & + & & + & & & + \\
\hline 92 Aglaia Lour. sp. & & + & & & & & 150 Melicope bonwickii (F.Muell.) T.G.Hartley & + & & & & & \\
\hline 93 Chisocheton patens Blume & + & & & & & + & 151 Melicope confusa (Merr.) T.S.Liu & + & & + & & & + \\
\hline 94 Chisocheton warburgii Harms & + & & & & & + & Sapindaceae & & & & & & \\
\hline 95 Dysoxylum acutangulum Miq. & & & & & & & 154 Guioa hirsuta Welzen & + & & & + & & + \\
\hline subsp. foveolatum (Radlk.) Mabb. & + & & & & + & & 155 Harpullia cupanioides Roxb. & + & & & & & \\
\hline 96 Dysoxylum densiflorum Miq. & + & & & & & & 156 Pometia pinnata J.R.Forst. \& G.Forst. & + & & & & & \\
\hline 97 Dysoxylum excelsum Blume & + & & + & & & & Sapotaceae & & & & & & \\
\hline 98 Dysoxylum nutans (Blume) Miq. & + & & & & & & 157 Palaquium luzoniense S.Vidal & + & & & & & + \\
\hline 99 Dysoxylum quadrangulatum Culmsee & & + & & & $+!$ & & 158 Pouteria firma (Miq.) Baehni & + & & + & & & + \\
\hline 100 Reinwardtiodendron Koord. sp. & & & & + & $(+)$ & & Staphyleaceae & & & & & & \\
\hline Meliosmaceae (Sabiaceae) & & & & & & & 159 Turpinia sphaerocarpa Hassk. & + & & & & & \\
\hline 101 Meliosma sumatrana Walp. & + & & & & & & Sterculiaceae & & & & & & \\
\hline Monimiaceae & & & & & & & 160 Sterculia macrophylla Vent. & + & & & & & \\
\hline 102 Kibara Endl. sp. & & + & & & & & 161 Sterculia oblongata R.Br. & + & & & + & & + \\
\hline 103 Levieria montana Becc. & + & & + & & + & & Theaceae & & & & & & \\
\hline 104 Matthaea sancta Blume & + & & & & + & & 162 Adinandra celebica Koord. & & & + & & & \\
\hline 105 Steganthera hirsuta Perkins & & & & + & + & & 163 Camellia lanceolata Seem. & & + & & & & \\
\hline Moraceae & & & & & & & 164 Eurya acuminata DC. & + & & + & & & \\
\hline 106 Artocarpus lacucha Buch.-Ham. & + & & & & + & & 165 Ternstroemia L.f. sp. & & & + & & & \\
\hline 107 Artocarpus teysmannii Miq. & & & & & & & Verbenaceae & & & & & & \\
\hline subsp. teysmannii & & + & & & & & 166 Vitex L. sp. & + & & & & & \\
\hline
\end{tabular}




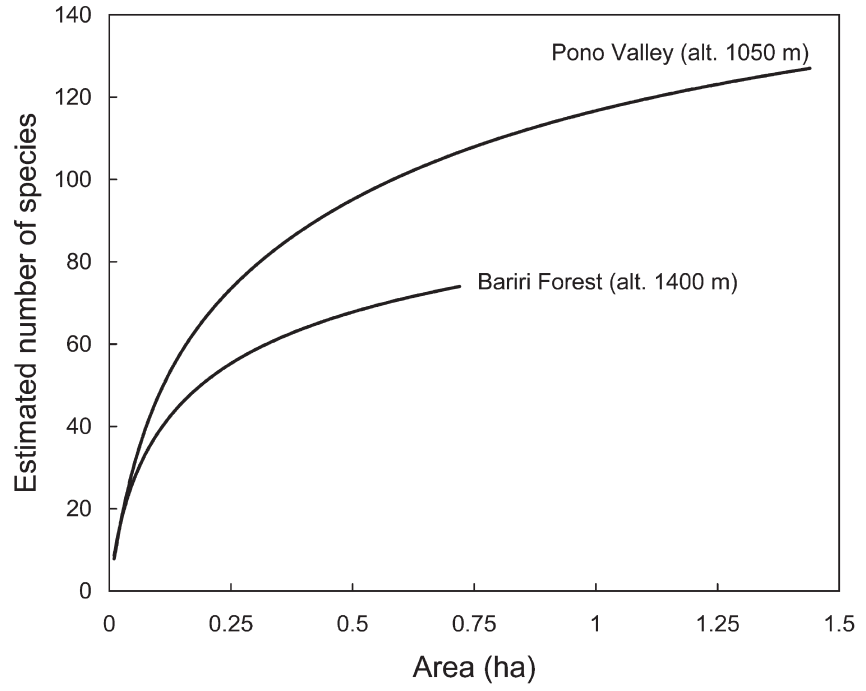

Fig. 1 Sample-based rarefaction curves for Pono Valley (alt. $1050 \mathrm{~m}$ ) and Bariri Forest (alt. $1400 \mathrm{~m}$ ).

difference in species richness is not an artefact. The samplebased rarefaction curve (Fig. 1) shows that already at half of the effectively sampled area in the Bariri Forest the curve starts rising slowly. If the curve is extrapolated, it reaches \pm 85 taxa at 1.44 ha. In contrast, the number of species is expected to exceed 100 already at 0.72 ha in Pono Valley.

The combined Pono Valley and Bariri Forest species list (Table 2) comprises a total of 166 taxa assigned to 49 families. Less than $1 \%$ of the sampled trees remained unidentified. At Pono Valley, the trees of $\mathrm{dbh} \geq 10 \mathrm{~cm}$ include 104 taxa with an additional 19 supplementary species found among the trees of $\mathrm{dbh} 2-9.9 \mathrm{~cm}$. The Bariri Forest comprises 60 tree species of $\mathrm{dbh} \geq 10 \mathrm{~cm}$ with 14 taxa additionally found among the trees of dbh 2-9.9 $\mathrm{cm}$. The two sites have 33 species in common. The highest number of species is found in Lauraceae (19 spp.), Euphorbiaceae (13 spp.), Elaeocarpaceae (12 spp.), Meliaceae (12 spp.), Rubiaceae (10 spp.), Moraceae (9 spp.) and Myrtaceae (9 spp.).

A total of $50 \%$ are found to be new records, with $19 \%$ as new records for Sulawesi (32 spp.) and $31 \%$ as new to Central
Sulawesi (51 spp.). The new records for Sulawesi include on family level the Gesneriaceae and the Hamamelidaceae. Tree ferns are explicitly not included in the checklist (Keßler et al. 2002), but are included in the present list. One new species was described based on the collections from Pono Valley (Culmsee 2008).

\section{Community composition}

The sub-montane and the lower montane forests have ten important tree families in common (based on FIV, Table 3). In Pono Valley, the Lauraceae have the highest importance (FIV = 30.2), closely followed by the Fagaceae, Sapotaceae, Moraceae and Euphorbiaceae. In the Bariri Forest, the Fagaceae are by far the most dominant family (FIV = 71.9). They are followed by the Myrtaceae, Elaeocarpaceae and Lauraceae.

The families Juglandaceae, Oleaceae and Theaceae have high importance in the lower montane forest, but their importance is low in the sub-montane forest. In contrast, the Asteraceae, Meliaceae, Myristicaceae, Rubiaceae and Rutaceae are important families in the sub-montane forest, but their importance decreases considerably in the lower montane forest. The Annonaceae, Cyatheaceae and Dracaenaceae are present in the sub-montane forest, but they are absent in the lower montane forest.

\section{DISCUSSION}

The large number of new records of tree species for the island of Sulawesi or its Central province is remarkable, in particular as the Lore Lindu National Park is among the parts of Sulawesi for which the largest plant collections are available (Cannon et al. 2007). For the whole island, the plant collection density is one of the lowest in Malesia (Roos et al. 2004), with 25 per 100 $\mathrm{km}^{2}$. Furthermore, only few plant collections are available from the quite extensive forests in good or old-growth conditions in other parts of Sulawesi (Cannon et al. 2007). That leads to the assumption that the relative number of new records or even new species could potentially be even higher in primary forests of other, less investigated parts of Sulawesi.

Compared to plot-based studies at similar altitudes in Malesia (Table 4), the plots in the present study are less species

Table 3 Family Importance Value (FIV; R: within-site ranking of families sorted by FIV), number of species (\# sp), relative frequency in \% (Rel FQ) and basal area $\left(\mathrm{BA} \mathrm{ha}^{-1}\right)$ for the most important tree families (FIV $\geq 5.0$ ) at Pono Valley (alt. $1050 \mathrm{~m}$ ) and Bariri Forest (alt. $1400 \mathrm{~m}$ ) based on trees dbh $\geq 10 \mathrm{~cm}$.

\begin{tabular}{|c|c|c|c|c|c|c|c|c|c|c|c|}
\hline \multirow[b]{2}{*}{$\mathrm{R}$} & \multicolumn{5}{|c|}{ Pono Valley } & \multicolumn{6}{|c|}{ Bariri Forest } \\
\hline & Family & FIV & $\# \mathrm{sp}$ & Rel FQ & $\mathrm{BA} \mathrm{ha}^{-1}$ & $\mathrm{R}$ & Family & FIV & $\# \mathrm{sp}$ & Rel FQ & BA ha-1 \\
\hline 2 & Fagaceae & 29.9 & 2 & 8.9 & 6.8 & 1 & Fagaceae & 71.9 & 5 & 15.9 & 17.8 \\
\hline 7 & Myrtaceae & 15.1 & 7 & 5.3 & 1.2 & 2 & Myrtaceae & 27.4 & 5 & 12.0 & 2.8 \\
\hline 3 & Sapotaceae & 26.4 & 2 & 8.0 & 5.9 & 9 & Sapotaceae & 10.8 & 1 & 3.1 & 2.3 \\
\hline 10 & Elaeocarpaceae & 9.8 & 5 & 2.5 & 1.0 & 3 & Elaeocarpaceae & 26.5 & 7 & 10.7 & 1.8 \\
\hline 5 & Euphorbiaceae & 21.1 & 12 & 7.0 & 1.1 & 7 & Euphorbiaceae & 11.4 & 4 & 4.2 & 0.3 \\
\hline 13 & Burseraceae & 8.4 & 2 & 3.7 & 1.0 & 5 & Burseraceae & 18.0 & 1 & 5.5 & 4.0 \\
\hline 12 & Icacinaceae & 9.2 & 1 & 5.4 & 1.0 & 10 & Icacinaceae & 9.0 & 1 & 4.7 & 1.0 \\
\hline 17 & Clusiaceae & 5.5 & 4 & 0.7 & 0.4 & 8 & Clusiaceae & 11.3 & 1 & 6.3 & 1.3 \\
\hline 28 & Oleaceae & 2.4 & 2 & 0.4 & 0.1 & 6 & Oleaceae & 17.3 & 1 & 12.5 & 1.2 \\
\hline 30 & Juglandaceae & 2.2 & 1 & 0.4 & 0.3 & 13 & Juglandaceae & 5.4 & 2 & 1.6 & 0.3 \\
\hline 38 & Theaceae & 1.4 & 1 & 0.3 & $<0.1$ & 11 & Theaceae & 6.1 & 3 & 1.0 & 0.1 \\
\hline 8 & Meliaceae & 15.9 & 9 & 4.8 & 1.0 & 27 & Meliaceae & 2.2 & 1 & 0.5 & $<0.1$ \\
\hline 11 & Rubiaceae & 9.7 & 7 & 2.6 & 0.3 & 17 & Rubiaceae & 3.8 & 1 & 1.6 & 0.2 \\
\hline 14 & Rutaceae & 6.6 & 3 & 2.9 & 0.3 & 16 & Rutaceae & 3.9 & 2 & 0.5 & 0.1 \\
\hline 15 & Asteraceae & 6.0 & 1 & 2.9 & 0.8 & 30 & Asteraceae & 2.0 & 1 & 0.3 & 0.1 \\
\hline 9 & Annonaceae & 9.9 & 5 & 3.8 & 0.6 & & & & & & \\
\hline 16 & Dracaenaceae & 5.6 & 1 & 3.5 & 0.4 & & & & & & \\
\hline 18 & Cyatheaceae & 5.4 & 1 & 3.7 & 0.3 & & & & & & \\
\hline
\end{tabular}


Table 4 Number of species (\# sp), basal area (BA) and top-3 families in this study compared to primary forest plots in Malesia available from literature (CEL = Sulawesi, BOR = Borneo, $\mathrm{PNG}=$ Papua New Guinea). Considered are trees of $\mathrm{dbh} \geq 10 \mathrm{~cm}$. The ranking of the top-3 families are based on Family Importance Value (FIV) for CEL Pono, CEL Bariri, CEL LLNP and PNG, respectively relative basal area (\%) for BOR 07N and BOR 17N.

\begin{tabular}{|c|c|c|c|c|c|c|}
\hline Site & BOR 07N & PNG & CEL Pono & CEL LLNP & CEL Bariri & BOR $17 \mathrm{~N}$ \\
\hline Reference & Aiba \& Kitayama (1999) & Wright et al. (1997) & this study & Kessler et al. (2005) & this study & Aiba \& Kitayama (1999) \\
\hline Altitude (m) & 700 & 900 & 1050 & $1100-1200$ & 1400 & 1700 \\
\hline$\# \mathrm{sp}$ & 148 & 228 & 104 & c. 150 & 60 & 84 \\
\hline $\mathrm{BA}\left(\mathrm{m}^{2} \mathrm{ha}^{-1}\right)$ & 34.0 & 37.1 & 35.4 & 139.8 & 37.1 & 36.4 \\
\hline Top-3 families & $\begin{array}{l}\text { Dipterocarpaceae } \\
\text { Ixonanthaceae } \\
\text { Lauraceae }\end{array}$ & $\begin{array}{l}\text { Lauraceae } \\
\text { Myristicaceae } \\
\text { Moraceae }\end{array}$ & $\begin{array}{l}\text { Lauraceae } \\
\text { Fagaceae } \\
\text { Sapotaceae }\end{array}$ & $\begin{array}{l}\text { Meliaceae } \\
\text { Moraceae } \\
\text { Lauraceae }\end{array}$ & $\begin{array}{l}\text { Fagaceae } \\
\text { Myrtaceae } \\
\text { Elaeocarpaceae }\end{array}$ & $\begin{array}{l}\text { Myrtaceae } \\
\text { Fagaceae } \\
\text { Podocarpaceae }\end{array}$ \\
\hline
\end{tabular}

rich. But with about double the number of species compared to Pono Valley, only the one-hectare-plot from Papua New Guinea (Wright et al. 1997) is truly exceptional. The difference is considered to be caused by the history of the Papua New Guinean flora (Primack \& Corlett 2006, Wright et al. 1997). The decrease in number of species with higher elevation in the Bariri Forest compared to Pono Valley is in accordance with the results from the Mt Kinabalu altitudinal transect study (Aiba \& Kitayama 1999). The basal area recorded for both the Pono Valley and the Bariri Forest is in accordance with data from all sites except the study of Kessler et al. (2005) where a basal area was recorded that was more than three times as high as in other studies.

In terms of the top-3 families, all sub-montane forests have the Lauraceae as important family in common. However, the overall dominant Dipterocarpaceae of the Bornean sub-montane forest are not represented in the studies east of Wallace's line. In the composition of the top-10 most important families (FIV), the forest site surveyed by Kessler et al. (2005) seems to be more similar to the Papua New Guinean forest (Wright et al. 1997) than to the Pono Valley forest. The lower montane Bariri Forest and the Bornean forest at $1700 \mathrm{~m}$ altitude are best comparable in having their two most important families in common, the Fagaceae and the Myrtaceae.

Acknowledgements Field-work was kindly supported by the Collaborative Research Centre SFB 552 at the University of Göttingen, funded by the German Research Foundation (DFG). The visit to the National Herbarium of the Netherlands, University of Leiden branch was facilitated by courtesy of EU-SYNTHESYS grant NL-TAF 3317. H. Culmsee wants to thank specialists for their help in plant identification and discussions on difficult taxa, at Kew: M.J.E. Coode, and at Leiden: C.C. Berg, W.J.J.O. de Wilde, M.M.J. van Balgooy, P.C. van Welzen, D.J. Mabberley, P.J.A. Keßler, F. Adema, H.P. Nooteboom and W. Vink.

\section{REFERENCES}

Aiba S, Kitayama K. 1999. Structure, composition and species diversity in an altitude-substrate matrix of rain forest tree communities on Mount Kinabalu, Borneo. Plant Ecology 140: 139-157.

Aiba S, Takyu M, Kitayama K. 2005. Dynamics, productivity and species richness of tropical rainforests along elevational and edaphic gradients on Mount Kinabalu, Borneo. Ecological Research 20: 279-286.

Cannon CH, Summers M, Harting JR, Keßler PJA. 2007. Developing conservation priorities based on forest type, condition, and threats in a poorly known ecoregion: Sulawesi, Indonesia. Biotropica 39: 747-759.

Colwell RK. 2006. EstimateS: Statistical estimation of species richness and shared species from samples software and user's guide, v8. http://viceroy. eeb.uconn.edu/estimates.

Culmsee H. 2008. Dysoxylum quadrangulatum, and notes on Meliaceae in Sulawesi. Blumea 53: 602-606.

FAO. 2006. World reference base for soil resources 2006. A framework for international classification, correlation and communication. World Soil Resources Reports 103.

Gotelli NJ, Colwell RK. 2001. Quantifying biodiversity: procedures and pitfalls in the measurement and comparison of species richness. Ecology Letters 4: 379-391.

Kessler M, Keßler PJA, Gradstein SR, Bach K, Schmull M, Pitopang R. 2005. Tree diversity in primary forest and different land use systems in Central Sulawesi, Indonesia. Biodiversity and Conservation 14: 547-560.

Keßler PJA, Bos M, Sierra Daza SEC, Kop A, Willemse LPM, Pitopang R, Gradstein SR. 2002. Checklist of woody plants of Sulawesi, Indonesia. Blumea Supplement 14: 1-160.

Kitayama K. 1992. An altitudinal transect study of the vegetation on Mount Kinabalu, Borneo. Vegetatio 102: 149-171.

Mori SA, Boom BM, De Carvalho A, Dos Santos TS. 1983. Southern Bahian moist forests. The Botanical Review 49: 155-232.

Primack R, Corlett R. 2006. Tropical rain forests. An ecological and biogeographical comparison. Blackwell Publishing, USA.

Roos MC, Keßler PJA, Gradstein SR, Baas P. 2004. Species diversity and endemism of five major Malesian islands: diversity-area relationships. Journal of Biogeography 31: 1893-1908.

Wright DD, Jessen JH, Burke P, Gomez de Silva Garza H. 1997. Tree and liana enumeration and diversity on a one-hectare plot in Papua New Guinea. Biotropica 29: 250-260. 